\title{
Cuantificación de la eliminación urinaria de ácido acetilsalicílico en el humano
}

\author{
Quantification of the urinary elimination of acetylsalicylic acid in humans
}

\author{
José Arias-Rico ${ }^{a}$, Reyna C. Jiménez Sánchez, ${ }^{b}$ Osmar A. Jaramillo Morales ${ }^{c}$, \\ Olga R. Flores Chávez ${ }^{d}$, Karina Chávez-Rubio ${ }^{e}$, Flor I. Castillo Reyes ${ }^{f}$, \\ María F. Segura Soto ${ }^{g}$ y Beatriz. Medina Arellano ${ }^{h}$
}

\begin{abstract}
:
Salicylate acetic acid (ASA), is rapidly absorbed by the stomach and the upper part of the intestine, the maximum level in the salicylate plasma is between 60 and 90 minutes. After absorption as ASA, it is hydroxylated to acetic acid and salicylate by esterases in the tissues and blood, the effects of ASA on the cell are complex and the main mechanisms include the inhibition of the enzyme oxygen cycle and the concomitant blockade of both prostaglandins and synthesis of thromboxane. This paper aims to determine the elimination of ASA in the urine of healthy volunteers and the influence of the presentation of the drug and the gastrointestinal $\mathrm{pH}$ in its elimination. To meet the objective, six healthy volunteers were required to take $250 \mathrm{mg}$ of ASA in the two presentations of the drug accompanied by different drinks (juice of one, orange, efferent, milk, mango yogurt), a spectrophotometer was used in the samples of urine collected at different times after the administration of the drug and the salicylate content in each sample was determined, measuring the $\mathrm{pH}$ and the volume collected from each urine sample, A $2 \mathrm{ml}$ aliquot of the urine sample was taken, then $8 \mathrm{ml}$ of $0.1 \mathrm{~N}$ hydrochloric acid was added, stirred and filtered, $5 \mathrm{ml}$ of the filtrate and $1 \mathrm{ml}$ of the trinder relative were also taken. resulting in a variation in the volume of the urine and in the $\mathrm{pH}$ according to the pharmaceutical form and the previously ingested beverage. After carrying out the development of the practice in a successful way we were able to analyze and verify the results obtained, the purification of ASA could be expressed in $\mathrm{ml} / \mathrm{mm}$ and thus verify the importance of the presentation of the drugs in the pharmacokinetics and the influence of Gastrointestinal $\mathrm{pH}$ in healthy patients, concluding that the concentrations obtained from salicylate in each collection interval were normalized.
\end{abstract}

Keywords: Pharmaceutical, absorption, active substance, acetylsalicylic acid, pharmacokinetics.

\footnotetext{
a Autor de Correspondencia, Universidad Autónoma del Estado de Hidalgo. Instituto de Ciencias de la Salud, https://orcid.org/0000-0003-0219-0410,Email: jose_arias@uaeh.edu.mx

b Universidad Autónoma del Estado de Hidalgo. Instituto de Ciencias de la Salud, https://orcid.org/0000-0001-9264-8514,

Email: crsitiji@hotmail.com

Universidad Autónoma del Estado de Hidalgo. Instituto de Ciencias de la Salud,

https://orcid.org/0000-0002-5325-3760,Email: oajmorales@gmail.com

d. Universidad Autónoma del Estado de Hidalgo. Instituto de Ciencias de la Salud, https://orcid.org/0000-0001-5719-1854, Email: ofloresc@uaeh.edu.mx

e. Universidad Autónoma del Estado de Hidalgo. Instituto de Ciencias de la Salud, http://orcid.org/0000-0001-5719-1854, Email: kary070895@gmail.com

f. Universidad Autónoma del Estado de Hidalgo. Instituto de Ciencias de la Salud, https://orcid.org/0000-0003-1784-3007, Email: ca415337@uaeh.edu.mx

g. Universidad Autónoma del Estado de Hidalgo. Instituto de Ciencias de la Salud, https://orcid.org/0000-0003-4172-928X,

Email: se316039@uaeh.edu.mx

h. Universidad Autónoma del Estado de Hidalgo. Instituto de Ciencias de la Salud, https://orcid.org/0000-0001-7440-0160,

Email:dymetrix1@gmail.com
} 


\section{Resumen}

El ácido acético del salicilato (AAS), es rápidamente absorbido por el estómago y la parte superior del intestino, el máximo nivel en el plasma de salicilato es entre 60 y 90 minutos. Después de su absorción como AAS se hidroxila a ácido acético y salicilato por esterasas en los tejidos y la sangre, los efectos del AAS sobre la célula son complejos y los principales mecanismos incluyen la inhibición de la enzima ciclo-oxigenasa y el concomitante bloqueo de ambos prostaglandinas y síntesis de tromboxano. El presente trabajo tiene como objetivo determinar la eliminación de AAS en la orina de voluntarios sanos y la influencia que tiene la presentación del fármaco y el pH gastrointestinal en su eliminación. Para cumplir el objetivo se requirió de seis voluntarios sanos que tomaran $250 \mathrm{mg}$ de AAS en las dos presentaciones del fármaco acompañados con diferentes bebidas (jugo de uva, naranja, eferente, leche, yogurt de mango), se utilizó de un espectrofotómetro en las muestras de orina recolectadas a diferentes tiempos posteriores a la administración del medicamento y se determinó el contenido del salicilato en cada una de las muestras, midiendo el $\mathrm{pH}$ y el volumen recolectado de cada muestra de orina. Se tomó una alícuota de $2 \mathrm{ml}$ de la muestra de orina, después se añadió $8 \mathrm{ml}$ de ácido clorhídrico $0.1 \mathrm{~N}$, Se agito y se filtró, también se tomó $5 \mathrm{ml}$ del filtrado y acciones $1 \mathrm{ml}$ del reactivo de trinder. dando como resultado una variación en el volumen de la orina y en el pH de acuerdo con la forma farmacéutica y la bebida previamente ingerida. Después de llevar a cabo el desarrollo de la práctica de manera exitosa pudimos analizar y verificar los resultados obtenidos, se pudo expresar en $\mathrm{ml} / \mathrm{mm}$ la depuración de AAS y así comprobar la importancia de la presentación de los medicamentos en la farmacocinética y la influencia de $\mathrm{pH}$ gastrointestinal en pacientes sanos, concluyendo que las concentraciones obtenidas de salicilato en cada intervalo de recolección fueron normalizándose.

Palabras Clave: Farmacéutica, absorción, principio activo, ácido acetilsalicílico, farmacocinética

\section{Introducción}

Los medicamentos no permanecen indefinidamente en el organismo. Existen procesos para eliminarlos y para terminar con su actividad biológica, principalmente la biotransformación y la excreción. Si se conocen las características fisicoquímicas de un fármaco y las propias de los comportamientos, corporales, se puede predecir en cierta medida el comportamiento emético de un fármaco en cuanto a su absorción, distribución y biotransformación y/o excreción. El riñón es uno de los órganos que llevan a cabo la eliminación de productos endógenos que desecha el organismo y también es para sustancias exógenas incluyendo a los fármacos o sus metabolitos. Lo anterior señala que la mayoría de los fármacos se eliminan con la orina, en mayor proporción que por otras vías. Los mecanismos involucrados en tales procesos comprenden: a) la filtración, si las moléculas del fármaco no son muy grandes y no están unidas a las proteínas no filtrables del plasma y b) la secreción activa y la pasiva de fármacos o de sus metabolitos desde el plasma a la orina tubular; sin embargo, la reabsorción de productos con alto coeficiente de participación lípido-agua limitan cuantitativamente la eliminación urinaria de numerosas substancias. (Córdova, I., Roxana, B., \& Galván Espejo, P. M. 2017).

Los compuestos polares y la variedad, y única de los fármacos no son capaces de difundir de regreso al plasma y por lo tanto se con la orina, a menos que se reabsorban por un mecanismo de transporte activo o intercambio, por consiguiente, la manipulación farmacológica internación del pH urinario con fármacos o acidificantes influye sobre los parámetros de la eliminación renal de numerosos fármacos de naturaleza acida o básica. En la mayoría de los casos, los mecanismos de eliminación renal tienen como propósito retirar una fracción constante del fármaco desde el plasma y en términos cuantitativos se expresa como una depuración o "limpia" de un cierto volumen de plasma por minuto. Si se conoce la concentración del fármaco en el plasma y en la orina, el volumen urinario y el tiempo de la colección de la orina, es posible expresar en ML/MN la depuración de un fármaco en mililitros por minuto. (Dominguez Mielgarejo \& Arias Rico, 2019). El objetivo fue determinar la eliminación de AAS en orina de voluntarios sanos.

\section{Metodología}

Al ser una investigación de naturaleza cuantitativa se procede a buscar personas sanas que estén dispuestas a colaborar con dicha investigación.

1. Quien participó como sujeto voluntario por mesa de trabajo, no debió haber recibido medicamento alguno por lo menos 48 horas antes del experimento y no debió tener antecedentes de hipersensibilidad al AAS o a otros fármacos o problemas de sangrado gastrointestinal.

2. En un recipiente limpio antes de la ingesta del medicamento. 
3. Los voluntarios tomaron $400 \mathrm{ml}$ de agua una hora antes del experimento.

4. Los voluntarios de las mesas 1 y 3 tomaron $250 \mathrm{mg}$ de AAS con $250 \mathrm{ml}$ de agua. Los voluntarios de las mesas 4,5 y 6 tomaron $250 \mathrm{mg}$ de AAS efervescentes con 250 de agua.

5. Se colectaron muestras de orina a los 20, 40. 80 y 120 minutos posteriores a la administración del medicamento y se determinó el contenido del salicilato en cada una de las muestras de acuerdo al siguiente procedimiento: (Imagen 1)

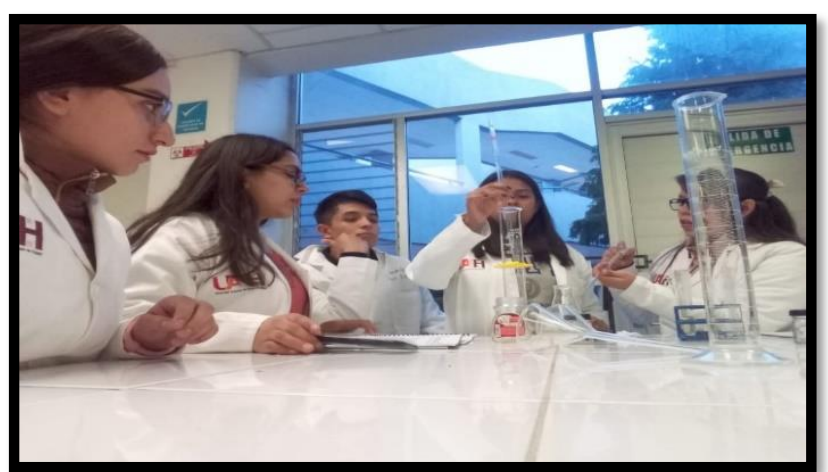

Imagen 1. Alumnos de la licenciatura en enfermería midiendo el volumen recolectado de cada muestra de orina

Se midió el $\mathrm{pH}$ y el volumen recolectado de cada muestra de orina, se tomó una alícuota de $2 \mathrm{ml}$ de la muestra de orina, se añadió $8 \mathrm{ml}$ de ácido clorhídrico $0.1 \mathrm{~N}$, se agito y se filtró, se tomó $5 \mathrm{ml}$ del filtrado y acciones $1 \mathrm{ml}$ del relativo de trinder.

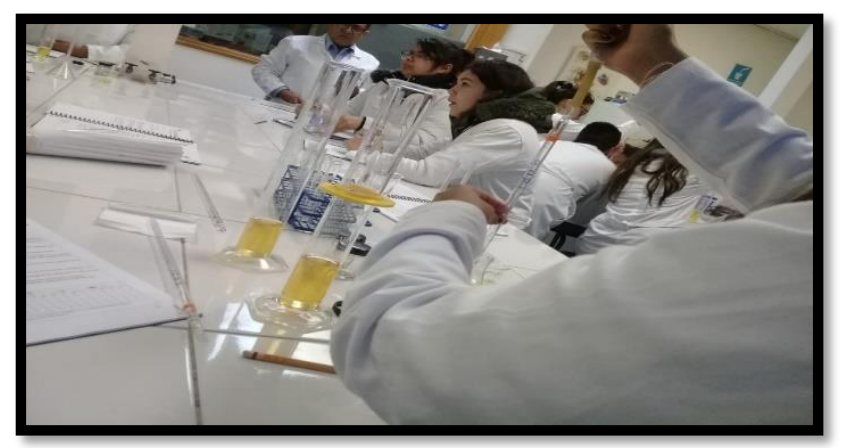

Imagen 2. Alumnos de la licenciatura en enfermería realizando medidas de $\mathrm{pH}$ en la orina recolectada.

6. En todos los casos se anotó el tipo de forma farmacéutica y la hora de ingestión de la misma en la tabla, calibre el espectrofotómetro con la muestra procesada da la orina basal, ajustando a 0 absorbancia. Agitaron y leyeron las muestras en el espectrofotómetro a una longitud de onda de $54^{\circ}$ con letra minúscula.

Se anotó en la tabla las lecturas obtenidas.

Interpone en la curva de calibración las lecturas obtenidas para cada muestra efectúe las correcciones para las diluciones realizadas y exprese el contenido del salicilato en un $\mathrm{ug} / \mathrm{ml}$ : de orina.

Para la estadística se hicieron tablas con los datos que obtuvimos de los voluntarios sanos con los diferentes alimentos líquidos que tomaron.

\section{Resultados}

La práctica de laboratorio es de suma importancia para los alumnos de enfermería de la Universidad Autónoma del Estado de Hidalgo para su formación perfeccionar en el manejo de administración de medicamentos llevando a la práctica real la farmacocinética de los medicamentos, comprobando la teoría y evidenciando la práctica se fortalece lo aprendido y el conocimientos, con ello adquiriendo las habilidades requeridas en el campo de la administración de medicamentos además de antes poder aplicarla llevan conocimiento teóricos suficientes para poder saber la importancia de cada práctica. Así los alumnos comienzan a conocer las formas de excreción en este caso se cuantifica la eliminación urinaria con una previa dosis de ácido acetilsalicílico acompañado de líquidos como jugo de una, naranja, eferente, leche, yogurt de mango. Donde cambiaron muchos los resultados de acuerdo a lo que cada alumno se tomó, si lo tomo en ayunas o durante la practica estando tomando. Mostrando un $\mathrm{pH}$ alto o bajo. Al inicio obteniendo dosis cerca de $300 \mathrm{ml}$ teniendo como volumen más bajo aspirina/jugo de mango, y un $\mathrm{pH}$ de 6 así mismo siendo más bajo aspirina/jugo de mango. (Tabla 1)

\begin{tabular}{|c|c|c|c|c|c|c|c|c|c|c|c|}
\hline \multirow{3}{*}{ MESA } & \multirow{3}{*}{ PRESENTACION } & \multicolumn{10}{|c|}{ COLECCIÓN DE ORINA } \\
\hline & & \multicolumn{2}{|r|}{0} & \multicolumn{2}{|c|}{20} & \multicolumn{2}{|c|}{40} & \multicolumn{2}{|c|}{80} & \multicolumn{2}{|c|}{120} \\
\hline & & & Vol & $\mathrm{PH}$ & Vol & PH & Vol & $\mathrm{PH}$ & Vol & $\mathrm{PH}$ & Vol \\
\hline 1 & Aspirina/Jugo de Uva & 6 & $300 \mathrm{ml}$ & 6 & $88 \mathrm{ml}$ & 6 & $250 \mathrm{ml}$ & 6 & 290 & 7 & $28 \mathrm{ml}$ \\
\hline 2 & Agua/Jugo de naranja & 7 & $248 \mathrm{ml}$ & 7 & $72 \mathrm{ml}$ & 7 & $225 \mathrm{ml}$ & 9 & 160 & 8 & 179 \\
\hline 3 & Aspirina efervecente/viña & 6 & $305 \mathrm{ml}$ & 6 & $68 \mathrm{ml}$ & 5 & 94 & 7 & 387 & 8 & 210 \\
\hline 4 & Leche & 6 & $88 \mathrm{ml}$ & 6 & $82 \mathrm{ml}$ & 7 & 70 & 7 & 70 & 8 & 130 \\
\hline 5 & Yogurt & 6 & $72 \mathrm{ml}$ & 6 & $34 \mathrm{ml}$ & 6 & 10 & 9 & 160 & 8 & 180 \\
\hline 6 & Aspirina/Jugo de mango & 5 & $60 \mathrm{ml}$ & 6 & $72 \mathrm{ml}$ & 6 & 10 & 7 & 36 & 7 & 39 \\
\hline
\end{tabular}

Tabla 1. Resultados de la práctica de laboratorio Del pH y VOLUMEN.

Como resultado final se obtuvo un volumen máximo de $210 \mathrm{ml}$ ahora siendo más bajo aspirina/ jugo de uva, y un $\mathrm{pH}$ de 8 . (Tabla 1)

Con los resultados de la Tabla 1, realizamos 3 graficas en donde los resultados se ven por separado y más claros, describiéndolas a continuación.

Nuestros resultados mostraron lo de ambos que son del $\mathrm{pH}$ y VOLUMEN, en esta combinamos ambos 
resultados del cómo fueron haciendo los cambios cada alimento líquido que los voluntarios consumieron. (Grafica 1)

En esta grafica nos está mostrando solamente los resultados del $\mathrm{pH}$ de los voluntarios que realizaron la práctica, muestra como el $\mathrm{pH}$ se va elevando un poco más en la colección de orina, especialmente en la 80 y 120 , con el agua/jugo de naranja y yogurt. (Grafica 2 )

Nuestro resultado de esta última prueba es del VOLUMEN de los voluntarios, mostrándonos un VOLUMEN variado a las 5 tomas de orina que se realizó, por ejemplo: la aspirina con jugo de uva y viña estuvieron elevadas en la toma 0 y 80 teniendo más de $300 \mathrm{ml}$, la aspirina con jugo de mago siempre tuvo resultado muy bajo no excediendo los $60 \mathrm{ml}$. (Grafica 3)

\section{Discusiones}

La presentación de medicamentos se ve afectando en la liberación y absorción de ello y esto depende su tiempo de vida del fármaco.

Lo que vamos a obtener de nuestras respuestas farmacológicas llegan a ser modificadas en función de la vía en la nosotros administramos, en este caso vía oral. La transformación va consistir como en un simple retraso en la acción, y en otros casos puede cambiar o anular totalmente el efecto, o inclusive llegar a alterarlo. Dándonos origen a nuevas moléculas que no necesariamente van a presentar la misma acción o van a seguir la misma farmacocinética.

La eliminación del fármaco nos hace comprender como se determina el fármaco, que en este caso hablamos del ácido acetilsalicílico, el cómo es cuantificado mediante la orina la cantidad de salicilatos totales excretados durante la administración.

El AAS se absorbe rápidamente cuando se administra por vía oral, se une a las proteínas del plasma y se distribuye ampliamente en el organismo; se metaboliza principalmente e nivel hepático, dando lugar a metabolitos que se eliminan por vía renal junto con la fracción AAS que no se metabolizo. Es importante señalar la influencia de los $\mathrm{PH}$ gastrointestinal y de la orina sobre la velocidad de absorción y eliminación del medicamento, respectivamente.

\section{Conclusión}

Las concentraciones obtenidas de salicilato en cada intervalo de recolección se multiplicaron por volumen de orina emitida en el mismo para obtener la cantidad acumulada excretada a lo largo de 72 horas. Estas cantidades fueron normalizadas por las dosis administradas expresadas en AAS, a fin de obtener el porcentaje acumulado de la dosis recuperada en orina a diferentes tiempos (\%R), muestra la velocidad de excreción urinaria en función del tiempo medio de cada intervalo de recolección. Se obtuvo el porcentaje acumulado de la recuperación de AAS en función del tiempo para cada formulación, partir de dichos datos se realizaron ajustes de las gráficas de los valores experimentales mediante análisis de región lineal.

\section{Referencias}

[1] Chaverri Jara1, R., Arce Jiménez, I., \& Valverde Zúñiga, A. (2016). Intoxicación Aguda Por Ácido. Revista Clínica De La Escuela De Medicina Ucr - Hsjd, 144-151.

[2] Zermeño, F. (2004). Acido Acetil Salicílico En El Tratamiento De Las Cefaleas. Archivos De Neurociencias (México, D.F.), 2.

[3] Brenner, G. M., \& Stevens, C. W. (2019). Farmacología Basica. España: Elsevier España, S.L.U.; Edición: 5.

[4] Brunton, L. L., Dandan, R. H., \& Knollmann, B. C. (2019). Goodman \& Gilman Las Bases Farmacologicas De La Terapéutica 13 A Edición. Ciudad De Mexico: Mc Graw Hill Education.

[5] Dominguez Mielgarejo, M., \& Arias Rico, J. (2019). Manual De Prácticas De Introducción A La Farmacologia. Pachuca, Hidalgo: Dgsa

[6] Rodriguez Peña, L. A. (20 De Mayo De 1994). Acta Farm. Bonaerense. Obtenido De Acta Farm. Bonaerense : Http://Www.Latamjpharm.Org/Trabajos/14/1/Lajop_14_1_1_2 5oyb45hyyo.Pdf

[7] Sepúlveda, R., Ortega, M., Donoso, N., \& Jara, A. (2018). Intoxicación Por Ácido Acetilsalicílico, Fisiopatología Y Manejo. Revista Médica De Chile. 
Publicación semestral, Educación y Salud Boletín Científico Instituto de Ciencias de la Salud Universidad Autónoma del Estado de Hidalgo, Vol. 8, No. 16 (2020) 158-162

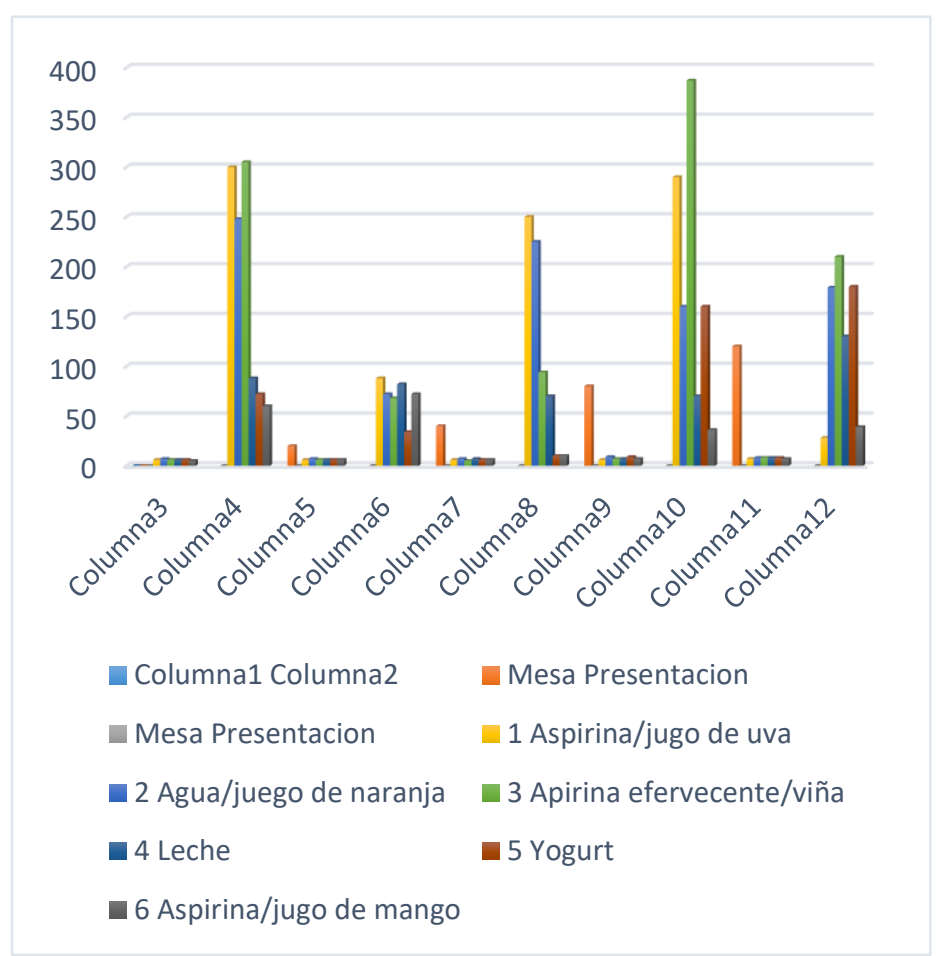

Grafica 1: Resultados totales de la práctica $7^{\circ}$ Cuantificación de la Eliminación urinaria de ácido acetilsalicílico.

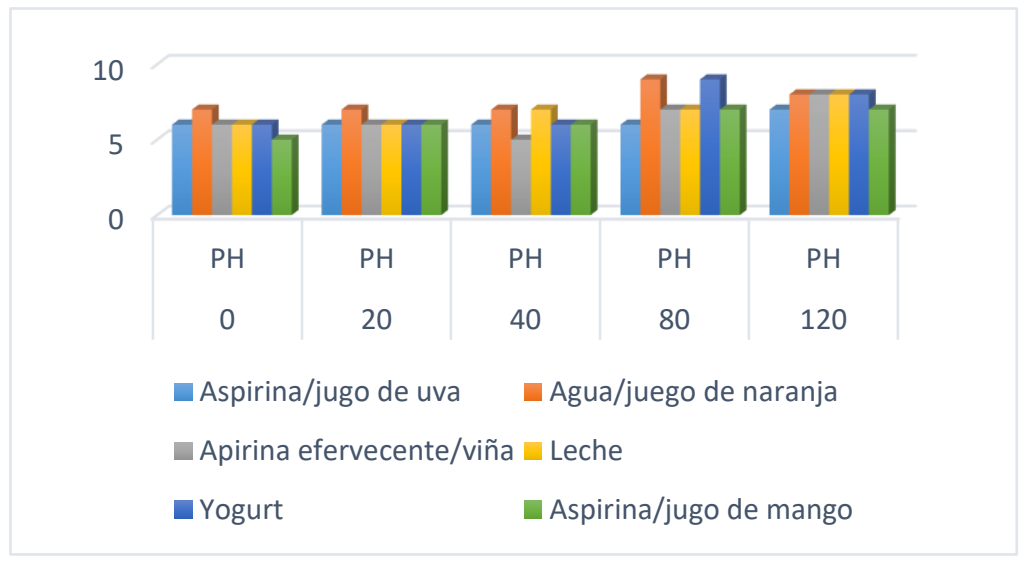

Grafica 2: Resultados totales del PH de la práctica $7^{\circ}$ Cuantificación de la Eliminación urinaria de ácido acetilsalicílico.

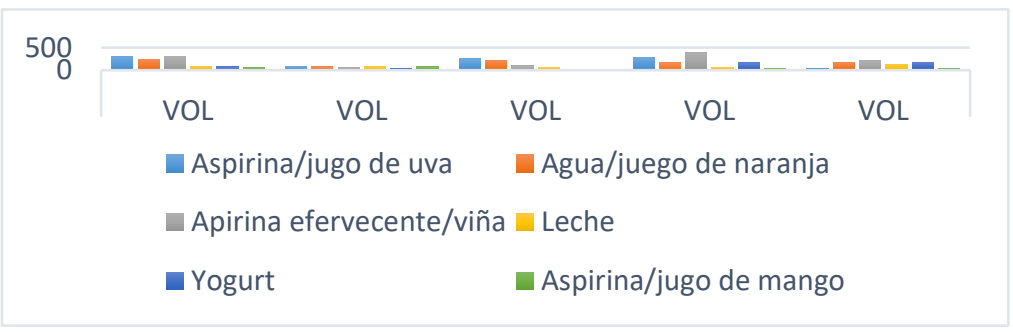

Grafica 3: Resultados totales del VOLUMEN de la práctica $7^{\circ}$ Cuantificación de la Eliminación urinaria de ácido acetilsalicílico. 